\title{
Re-exploration after off pump coronary artery bypass grafting: incidence, risk factors and impact of timing
}

\author{
Kartik Patel ${ }^{1}$, Sudhir Adalti ${ }^{1}$, Shreyas Runwal ${ }^{1}$, Rahul Singh ${ }^{1}$, Chandrasekaran \\ Ananthanarayanan ${ }^{2}$, Chirag Doshi ${ }^{1}$, and Himani Pandya ${ }^{1}$ \\ ${ }^{1}$ U.N.Mehta Institute of Cardiology and Research Center \\ ${ }^{2}$ U. N. Mehta Institute of Cardiology and Research Center
}

June 23, 2020

\begin{abstract}
Objective: Re-exploration after cardiac surgery still remained a troublesome complication. There is still scarcity of data about the effect of re-exploration after off-pump coronary arterial bypass grafting (OPCABG). We here represent our experience of re-exploration following OPCABG. Method: Total 5990 OPCABG were performed at our center, out-off these 132 (2.2\%) patients were re-explored in the OR and were included in this study. The medical records of these patients were retrospectively reviewed. Results: The most common cause of re-exploration was bleeding (83.3\%) and most common site of bleeding was from graft/anastomosis (53.8\%). Mean time to re-exploration was $9.75 \pm 8.65$ hours. 30-day mortality was $1.41 \%$. On univariate and multiple regression analysis, emergency surgery, preoperative low platelet count, and number of grafts were found to be an independent risk factor for re-exploration. On multiple regression, emergency surgery, euroscoreII, low platelet count, low ejection fraction, re-exploration, time to re-exploration, blood products used, high post-op serum creatinine and bilirubin, were found to be an independent factor $(\mathrm{p}<0.001)$ for mortality. On receiver-operating characteristic analysis, optimum cut off for time to re-exploration was 14 hours with sensitivity $81.3 \%$, specificity of $80 \%$ and area under curve of 0.798 . Patients who re-explored late ( $>14$ hour) had significantly high mortality (30.55\%vs7.3\%) and morbidity. Conclusion: Delaying the re-exploration is associated with three-fold increase in mortality and morbidity. So strategy of minimizing the incidence of re-exploration like use of minimally invasive surgery and early re-exploration with judicial use of products should be use to improve outcome after re-exploration following off-pump CABG.
\end{abstract}

\section{Introduction}

Post-operative hemorrhage requiring re-exploration after cardiac surgery, still remained a troublesome complication. There are reports from the various authors about the adverse outcome associated with the reexploration (1-6) while on the other hand, Tambe et al. believe that it is not associated with an adverse outcome (7). Nowadays due to advancement in cardiopulmonary bypass (CPB) technology and availability of point of care testing, the safety of cardiac surgery, in terms of bleeding, has been increased. However, many patients' related, intraoperative as well as post-operative unmodifiable factors are still persists which can contribute to the bleeding. Because of all these issues, risk of bleeding could not be eliminated completely till date (1- 4).

There are many concerns associated with the re-exploration. It has been found to be an independent factor for mortality as well as major morbidity $(1,5,6,8)$. Some authors also evaluated effect of timing of re-exploration on the adverse outcome; some authors reported better outcome with early re-exploration while others demonstrated no benefit from early re-exploration $(6,8-11)$. Re-exploration after coronary artery bypass surgery $(\mathrm{CABG})$, which still remain the standard treatment for most patients with left coronary artery disease and multivessel disease, is also associated with higher mortality and morbidity $(9,10)$. Now a days, the off-pump 
CABG (OPCABG) strategy has been tried to demonstrate with a lower rate of adverse effects, such as neurological complications by avoiding aortic manipulation and bleeding complications by avoiding complete heparinization and the inflammatory reactions resulting from cardiopulmonary bypass (12-14). Also there is still lack of data about the factors associated with re-exploration and mortality in the patients undergoing off pump coronary arterial bypass surgery (OPCABG). We here represent our Centre's experience regarding risk factors of the mortality and morbidity in patients underwent re-exploration following OPCABG.

\section{Patients and methods:}

Between January 2016 and December 2018, total 5990 OPCABG were performed in the department of the adult cardiac surgery at our center. One hundred and thirty two patients $(2.2 \%)$ were re-explored in the operation room (OR) and are included in our study. Patients explored for sternal wound infections, required graft revision $(\mathrm{n}=59)$ or required $\mathrm{CPB}$ during re-exploration were excluded from the study. The study was approved by our institutional ethics committee (UNMICRC/CVTS/2017/05) and written informed consent was waived off due to retrospective nature of the study. The medical records of these patients were retrospectively reviewed. The demographic data, preoperative data including preoperative use of antiplatelet, INR, creatinine etc, were noted. The intraoperative data of the main surgery as well as indication for reexploration, duration of surgery, estimated blood loss and intraoperative findings during the re-exploration were also noted. Post-operative data including hospital and intensive unit stay, morbidity and 30- day mortality were also noted.

\section{Management of antiplatelet and anticoagulation:}

It's our institutional policy to switch the patients to low dose aspirin $(75 \mathrm{mg})$ from dual antiplatelet at least 5 days before elective surgeries which is continued till the day of surgery. For off pump surgeries, heparin dose given is $1-2 \mathrm{mg} / \mathrm{kg}$ to maintain activated clotting time of $200-250$.

\section{Decision for re-exploration:}

The decision to perform re-exploration was left upon to the operating surgeon and was based on the standard criteria of Kirklin and Barratt-Boyes (15). During re-exploration, cause of bleeding was noted. Any surgical bleeding sites were controlled using stainless-steel clips, re-enforcing sutures, or electro-cautery. In cases of continuous and diffuse bleeding that could not be controlled surgically, the oozing area was packed with gauze, only skin was closed (sternum was not closed). After the re-exploration patient shifted to ICU and managed with standard protocols of the institute.

\section{Statistical analysis}

Statistical analysis was performed using SPSS software version 22. All data were expressed as mean \pm standard deviation or percentage as appropriate. To evaluate differences between groups, the unpaired student's $\mathrm{t}$ test for continuous variables and the chi-square test/fisher exact test for categorical variables was used, as appropriate. We calculated a receiver-operating characteristic (ROC) analysis and report sensitivity and specificity for time and re-exploration. The area under the ROC curve (AUC) was the overall performance measure of the accuracy of the test. A stepwise logistic regression was performed to evaluate independent markers associated with re-exploration and mortality. A p value of $<0.05$ (for a two-sided test) was considered significant.

\section{Results:}

There were $4536(75.72 \%)$ males. Mean age of the study population was $57.50+-8.53$ years. Most common diagnosis was triple vessel disease $(\mathrm{n}=4613,77 \%)$. Mean numbers of grafts inserted were $3+-0.83$. LIMA was used in 5972 patients (99.7\%). Most common grafting strategy was pedicle left internal mammary artery graft (LIMA) to left anterior descending artery (LAD) and saphenous vein to other territory. Total arterial revascularization in the form of LIMA (in situ, pedicle)-RIMA (skeletonized) or LIMA (in situ, pedicle)Radial 'Y' configuration was performed in 620 (10.35\%) patients. Emergency surgery was performed in 560 $(9.34 \%)$ patients and coronary endarterectomy performed in $886(14.8 \%)$ patients. Most common grafting 
strategy for endatrectomy vessel was direct anastomosis (of LIMA or saphenous vein) followed by grafting upon on lay patch of saphenous vein. Other demographic and preoperative variables are shown in Table 1.

Out of 5990 off pump coronary bypass surgeries, total $132(2.2 \%)$ patients were re-explored. The most common cause of re-exploration was bleeding $(\mathrm{n}=110,83.33 \%)$ followed by cardiac tamponade $(\mathrm{n}=15,11.36 \%)$ and unspecified $(\mathrm{n}=5,3.8 \%)$. The most common site of bleeding was from graft/anastomosis $(53.8 \%)$, followed by sternum including LIMA bed $(31.1 \%)$, others (10.6\%, pacing wire site, thymus etc.) and none (4.54\%). Mean time to re-exploration was 9.75+-8.65 hours (Median 5.5 hour, range 1-36 hour). Other intraoperative and postoperative variables are shown in Table 2.

We found that pre-operative low platelet count $(\mathrm{p}=0.000)$, emergency surgery $(\mathrm{p}=0.008)$, and number of grafts $(\mathrm{p}=0.000)$, were significant risk factors for re-exploration on univariate as well as multivariable analysis $\left(\mathrm{F}(4,594)=27.72, \mathrm{p}=0.000, \mathrm{R}^{2}=0.497\right)$ while age, sex, body surface area, diabetes, hypertension, peripheral vascular disease, pre-operative hemoglobin, pre-operative prothrombin time, pre-operative serum creatinine as well as serum bilirubin, pre-operative ejection fraction, total arterial revascularization, LIMA use and endarterectomy were not found risk factors for re-exploration after OPCABG ( $>>0.05$, Table 3,4$)$. Patient who re-explored had significantly increase morbidity in the form of increase drain output $(\mathrm{p}=0.00)$, number of blood product transfusion $(\mathrm{p}=0.00)$, more ICU stay $(\mathrm{p}=0.00)$, more ventilation time $(\mathrm{p}=0.00)$, more IABP use $(\mathrm{p}<0.001)$, renal dysfunction $(\mathrm{p}=0.002)$, deep sternal wound infection $(\mathrm{p}<0.001)$ and hospital stay $(\mathrm{p}=0.00)$.

30- Day mortality in the study population was $1.41 \%$. 30-day mortality was significantly more in patient who underwent re-exploration ( $13.63 \%$ vs $1.14 \%, \mathrm{p}=0.000)$. The most common cause of the death was sepsis $(66.6 \%)$ with multi organ dysfunction followed by low cardiac output syndrome. On multiple regression analysis (Table 4), preoperative platelet count, emergency surgery, euroscore II, number of grafts, preoperative ejection fraction, postoperative serum bilirubin and creatinine, no of blood products used, re-exploration and time to re-exploration found to be an independent risk factor for mortality $(\mathrm{F}(28,3076)=40.54, \mathrm{R} 2=0.519$, $\mathrm{p}=0.000$ for model fit) while age, sex, body surface area, previous MI, diabetes, hypertension, chronic obstructive pulmonary disease, preoperative prothrombin time, preoperative serum bilirubin, preoperative serum creatinine, total arterial coronary bypass grafting, presence of atrial fibrillation and performing coronary endarterectomies were not found statistically significant factors for mortality.

We analyzed effect of time delay on re-exploration because on multiple regression analysis B coefficient was highest for time to re-exploration. On receiver operating curve analysis, we found that the optimum cut off for time to re-exploration was of 14 hours with sensitivity $81.3 \%$ and specificity of $80 \%$ and AUC of 0.798 (figure 1).

As shown in Table 5, while comparing groups of patients who underwent re-exploration early (time to reexploration after completion of primary operation $<14$ hour as per cut-off) and delayed, later group had significantly high mortality ( $30.55 \%$ vs $7.29 \%, \mathrm{p}=0.000)$, higher drain output $(995.54+-380.2$ vs $1458.16+-$ $543.20, \mathrm{p}=0.00)$, higher number of blood products received (11.14+-11.89 vs $23.69+-10.09, \mathrm{p}=0.000)$, more frequently underwent coronary endarterectomy ( 6 vs12, $\mathrm{p}=0.000)$, higher incidence of tamponade ( 3 vs 12 , $\mathrm{p}=0.000)$, higher ventilation time $(13.05+-6.39$ vs $41.10+-81.08)$ and high ICU stay $(5.03+-3.74$ vs $8.53+-$ $6.03, \mathrm{p}=0.02$ ).

\section{Discussion :}

Post-operative hemorrhage requiring re-exploration after cardiac surgery still remains a troublesome complication with an incidence of $15 \%$ in the early days of cardiac surgery to $2-6 \%$ now a days $(5,6,9,10,16)$. There are many factors for reduction of the incidence of the re-exploration as well as blood transfusion out of which one of the factor is performing 'off - pump CABG' (OPCABG) for revascularization (12- 14). However, need for re-exploration was not totally alleviated by using off pump coronary artery revascularization strategies and there is still lake of data regarding incidence, risk factors and effect of timing of re-exploration after OPCABG 
The overall incidence of re-exploration in our cohort was $2.2 \%$ with most common site of the bleeding was graft branch/anastomosis (53.8\%). Karthik et al. and choong et al. $(9,10)$ also reported similar findings using on-pump CABG strategy predominantly. Thus we can conclude that whether we performed CABG with or without $\mathrm{CPB}$, most common site of the bleeding is from graft/anastomosis. They also reported incidence of diffuse ooze of around $25 \%$ while the same was $4.5 \%$ in our study. We believe that avoidance of CPB in our cohort is responsible for this findings highlighting another advantage of OPCABG over on-pump CABG.

We observed that emergency surgery, low platelet count as well as presence of thrombocytopenia and number of distal anastomosis, were found to be an independent factor for the re-exploration. Emergency surgery has been well documented as a cause for re-exploration and mortality $(10,17)$. One would expect this finding as they had received either loading doses of anti-aggregation therapy and/or continued use of dual antiplatelet, letting them more prone for bleeding. The influence of low preoperative platelet count on post-cardiac surgery bleeding is also well documented in literature $(18,19)$. Recently, one of the study, demonstrated that preoperative thrombocytopenia is associated with increased postoperative blood loos as well as increased risk of severe- massive bleeding after CABG (19). However, in this study, only $20 \%$ of patients underwent off pump CABG. On would easily assume that presence of thrombocytopenia will aggravate the effects of $\mathrm{CPB}$ on homeostasis and platelet dysfunction leading to increased risk of bleeding. However, this finding may not be applicable who are not exposed to deleterious effects of CPB. Rannuci. M et al showed that the platelet function linearly associated with platelet count (20). This explains association of platelet count with bleeding in patients undergoing OPCABG. We also found that more number of distal anastomosis was independently associated with re-exploration as documented by many authors $(10,16)$. This association can be explained by the finding that most common site of bleeding after CABG is from graft/anastomosis. Thus doing more number of grafts exposes more number of potential bleeding sites and increase the risk of bleeding.

The reported mortality of the patients undergoing re-exploration has been high in the range of 5.7 to $15.8 \%$ (3-8). In our cohort reported mortality was $13.6 \%$. Emergency surgery and higher euroscore are well documented cause of mortality after cardiac surgery $(9,17)$. One would expect this finding as these patients are generally sicker with unstable hemodynamics. Thrombocytopenia has been well documented as a risk factor for mortality in critically ill patient (21), but limited studies are available for post- cardiac surgery patients, however even these studies have documented thrombocytopenia as a bad prognostication factor as shown by us $(22,23)$. We previously showed that hyperbilirubinemia after cardiac surgery was known to increase mortality (24). Recent report suggests that once developed, it was associated with increased mortality irrespective of types of surgery (25).

Many authors have also found that re-exploration itself is an independent risk factor for mortality $(8,9,26)$. We have also seen that it independently increases the risk of mortality. Time after re-exploration has recently gained attention due to various authors reported poor outcome of the patients who underwent re-exploration late ( $>12$ hour) after cardiac surgery. We also observed similar findings in our study cohort. However, we have found that the optimum cut off value for re-exploration was 14 hour with AUC of around 0.8. On further analysis of these subgroup, we have found that patient who explored early (time to re-exploration after completion of primary operation $<14$ hour) had less mortality rate $(7.3 \%$ vs $30.55 \%)$.Similar finding have been documented by others $(9,10)$. Also incidence of endarterectomies, cardiac tamponade and drain output was more in the patients who explored late ( $>14$ hour) as compared to the patients who explored early. We routinely under corrected the heparin with protamine in a patient who underwent endarterectomies. This leads to continuous small amount of bleeding over the time requiring continuous transfusion of the blood products and delaying the re-exploration (as it failed to reach defined criteria). These small amount of blood often clots around heart especially around right atrium causing cardiac tamponade like feature leading to re-exploration. So we think that under-correction of heparin after OPCABG is a double-edged sword and it should be use very judiciously.

Blood transfusion after cardiac surgery has been found to be independent risk factor for mortality $(6,9$, 
10, 27) as well as post-operative infections. Murphy et al had also reported dose dependent relationship of blood products use with mortality and morbidity (27). We have observed that in patient who re-explored late receive more blood product as compared to patient who re-explored early. This probably also explained higher mortality in a patient who re-explored late.

We have also observed significantly less incidence of re-exploration in patients who underwent minimally invasive CABG (MIDCABG). MIDCABG as well as hybrid approach has been associated with less surgical trauma, less need for blood transfusion, less incidence of surgical site trauma, shorter hospital stay and faster recovery in carefully selected patients $(28,29)$. So one of the approach to decrease incidence of re-exploration is performing minimally invasive or hybrid surgery in appropriate patient.

\section{Limitation:}

The main limitation of the study was its retrospective nature. Another limitation is strict adherence to the criteria for re-exploration and decision of re-exploration was left open the operating surgeon so effect of bias cannot be ruled out.

\section{Conclusion:}

Re-exploration after off pump CABG still associated with high mortality and morbidity. Delaying the reexploration is associated with three-fold increase in mortality and morbidity. So strategy of minimizing the incidence of re-exploration like use of minimally invasive surgery and if required early re-exploration with judicial use of products should be use to improve outcome after re-exploration for off-pump CABG.

Conflicts of interest : None

\section{Sources of funding for the work : Nil}

Figure legends:

Figure 1: ROC curve of the study population showing optimum cut off time to re-exploration after surgery

1. Biancari F, Mikkola R, Heikkinen J, Lahtinen J, Airaksinen KEJ, Juvonen T. Estimating the risk of complications related to re-exploration for bleeding after adult cardiac surgery: a systematic review and meta-analysis. Eur J Cardiothorac Surg 2012; 41:50-5.

2. Vivacqua A, Koch CG, Yousuf AM, Nowicki ER, Houghtaling PL, Blackstone EH et al. Morbidity of bleeding after cardiac surgery: is it blood transfusion, reoperation for bleeding, or both? Ann Thorac Surg 2011; 91:1780-90.

3. Kristensen KL, Rauer LJ, Mortensen PE, Kjeldsen BJ. Reoperation for bleeding in cardiac surgery. Interact CardioVasc Thorac Surg 2012; 14: 709-13.

4. Haneya A, Diez C, Kolat P, Suesskind-Schwendi MV, Ried M, Schmid C et al. Re-exploration for bleeding or tamponade after cardiac surgery: impact of timing and indication on outcome. Thorac Cardiovasc Surg 2014; 63:51-7.

5. Ohmes LB, Di Franco A, Guy TS, Lau C, Munjal M, Debois W et al. Incidence, risk factors, and prognostic impact of re-exploration for bleeding after cardiac surgery: a retrospective cohort study. Int J Surg 2017; 48:166-73.

6. Ruel M, Chan V, Boodhwani M, McDonald B, Ni X, Gill G et al. How detrimental is reexploration for bleeding after cardiac surgery? J Thorac Cardiovasc Surg 2017; 154:927-35.

7. Tambe SP, Kimose H-H, Raben Greisen J, Jakobsen C-J. Reexploration due to bleeding is not associated with severe postoperative complications. Interact CardioVasc Thorac Surg 2017; 25: 233-40.

8. Jason M Ali, Kate Wallwork, Narain Moorjani. Do patients who require re-exploration for bleeding have inferior outcomes following cardiac surgery? Interact CardioVasc Thorac Surg 2019; 28:613-8.

9. Choong CK, Gerrard C, Goldsmith KA, Dunningham H, Vuylsteke A. Delayed re-exploration for bleeding after coronary artery bypass surgery results in adverse outcomes. Eur J Cardiothorac Surg $2007 ; 31: 834-8$. 
10. Karthik S, Grayson AD, McCarron EE, Pullan DM, Desmond MJ. Reexploration for bleeding after coronary artery bypass surgery: Risk factors, outcomes, and the effects of time delay. Ann Thoracic Surg 2004; 2:527-534.

11. Ranucci M, Bozzetti G, Ditta A, Cotza M, Carboni G, Ballotta A. Surgical reexploration after cardiac operations: why a worse outcome?. The Annals of thoracic surgery 2008; 86(5):1557-62.

12. Raja SG. Total arterial off-pump coronary revascularization: The Holy Grail?. Current Opinion in Cardiology. 2019 Sep 1; 34(5):552-6.

13. Benedetto U, Altman DG, Gerry S, Gray A, Lees B, Flather M, Taggart DP, Arterial Revascularization Trial Investigators. Off-pump versus on-pump coronary artery bypass grafting: Insights from the Arterial Revascularization Trial. The Journal of thoracic and cardiovascular surgery. 2018 Apr 1;155 (4):1545-53.

14. Puskas JD1, Williams WH, Duke PG, Staples JR, Glas KE, Marshall JJ, et al. Off-pump coronary artery bypass grafting provides complete revascularization with reduced myocardial injury, transfusion requirements, and length of stay: a prospective randomized comparison of two hundred unselected patients undergoing off-pump versus conventional coronary artery grafting. J Thorac Cardiovasc Surg 2003; 125:797-806.

15. Kirklin, J.W. and Barratt-Boyes, B. G. Cardiac Surgery, 2nd edn, Wiley, New York, 1993.

16. Dacey LJ, Munoz JJ, Baribeau YR, Johnson ER, Lahey SJ, Leavitt BJ, et al. Re-exploration for haemorrhage following coronary artery bypass grafting. Incidence and risk factors. Arch Surg 1998; $133: 442-7$.

17. Schumer EM, Chaney JH, Trivedi JR, Linsky PL, Williams ML, Slaughter MS. Emergency coronary artery bypass grafting: indications and outcomes from 2003 through 2013. Texas Heart Institute Journal 2016; 43(3):214-9.

18. Lopes CT, Brunori EF, Cavalcante AM, Moorhead SA, Swanson E, de Lima Lopes J, de Barros AL. Factors associated with excessive bleeding after cardiac surgery: A prospective cohort study. Heart \& Lung. 2016 Jan 1;45(1):64-9.

19. Nammas W, Dalen M, Rosato S, Gherli R, Reichart D, Gatti G, Onorati F, Faggian G, De Feo M, Bancone C, Chocron S. Impact of preoperative thrombocytopenia on the outcome after coronary artery bypass grafting. Platelets 2019 May 19;30(4):480-6.

20. Ranucci M, Baryshnikova E. The interaction between preoperative platelet count and function and its relationship with postoperative bleeding in cardiac surgery. Platelets 2017 Nov 17;28(8):794-827.

21. Williamson, DR, Albert, M, Heels-Ansdell, D, Arnold, DM, Lauzier, F, Zarychanski, R, et al. PROTECT Collaborators; Canadian Critical Care Trials Group; Australian and New Zealand Intensive Care Society Clinical Trials Group, Thrombocytopenia in critically ill patients receiving thromboprophylaxis: Frequency, risk factors, and outcomes. Chest (2013) ;144:1207-15.

22. Kertai MD, Zhou S, Karhausen JA, Cooter M, Jooste E, Li YJ, et al. Platelet counts, acute kidney injury, and mortality after coronary artery bypass grafting surgery. The Journal of the American Society of Anesthesiologists 2016; 124(2):339-52.

23. Matthias Thielmann, Marlene Bunschkowski, Paschalis Tossios, Sixten Selleng, Gunter Marggraf, Andreas Greinacher ,et al. Perioperative thrombocytopenia in cardiac surgical patients - incidence of heparin-induced thrombocytopenia, morbidities and mortality. Eur J Cardiothorac Surg 2010; 37(6):1391-5.

24. Sharma P, Ananthanarayanan C, Vaidhya N, Malhotra A, Shah K, Sharma R. Hyperbilirubinemia after cardiac surgery: An observational study. Asian Cardiovascular and Thoracic Annals 2015 Nov;23(9):1039-43.

25. Farag M, Veres G, Szabo G, Ruhparwar A, Karck M, Arif R. Hyperbilirubinaemia after cardiac surgery: the point of no return. ESC heart failure 2019 Aug;6(4):694-700.

26. Unsworth-White MJ, Herriot A, Valencia O, Poloniecki J, Smith EJ, Murday AJ, Parker DJ, Treasure T. Resternotomy for bleeding after cardiac operation: a marker for increased morbidity and mortality. The Annals of thoracic surgery 1995 Mar 1;59(3):664-7.

27. Gavin J. Murphy, Barnaby C. Reeves, Chris A. Rogers, Syed I.A. Rizvi, Lucy Culliford , and Gianni 
D. Angel. Increased Mortality, Postoperative Morbidity, and Cost After Red Blood Cell Transfusion in Patients Having Cardiac Surgery. Circulation 2007; 116:000-000.

28. Rodriguez M, Ruel M. Minimally invasive multivessel coronary surgery and hybrid coronary revascularization: can we routinely achieve less invasive coronary surgery?. Methodist DeBakey cardiovascular journal. 2016 Jan;12(1):14.

29. Guida GA, Guida GA, Bruno VD, Zakkar M, De Garate E, Pecchinenda MT, Homes A, Borzellino C, Mendoza P, Pecora G, Bonillo I. Left thoracotomy approach for off-pump coronary artery bypass grafting surgery: 15 years of experience in 2500 consecutive patients. European Journal of CardioThoracic Surgery. 2020 Feb 1;57(2):271-6.

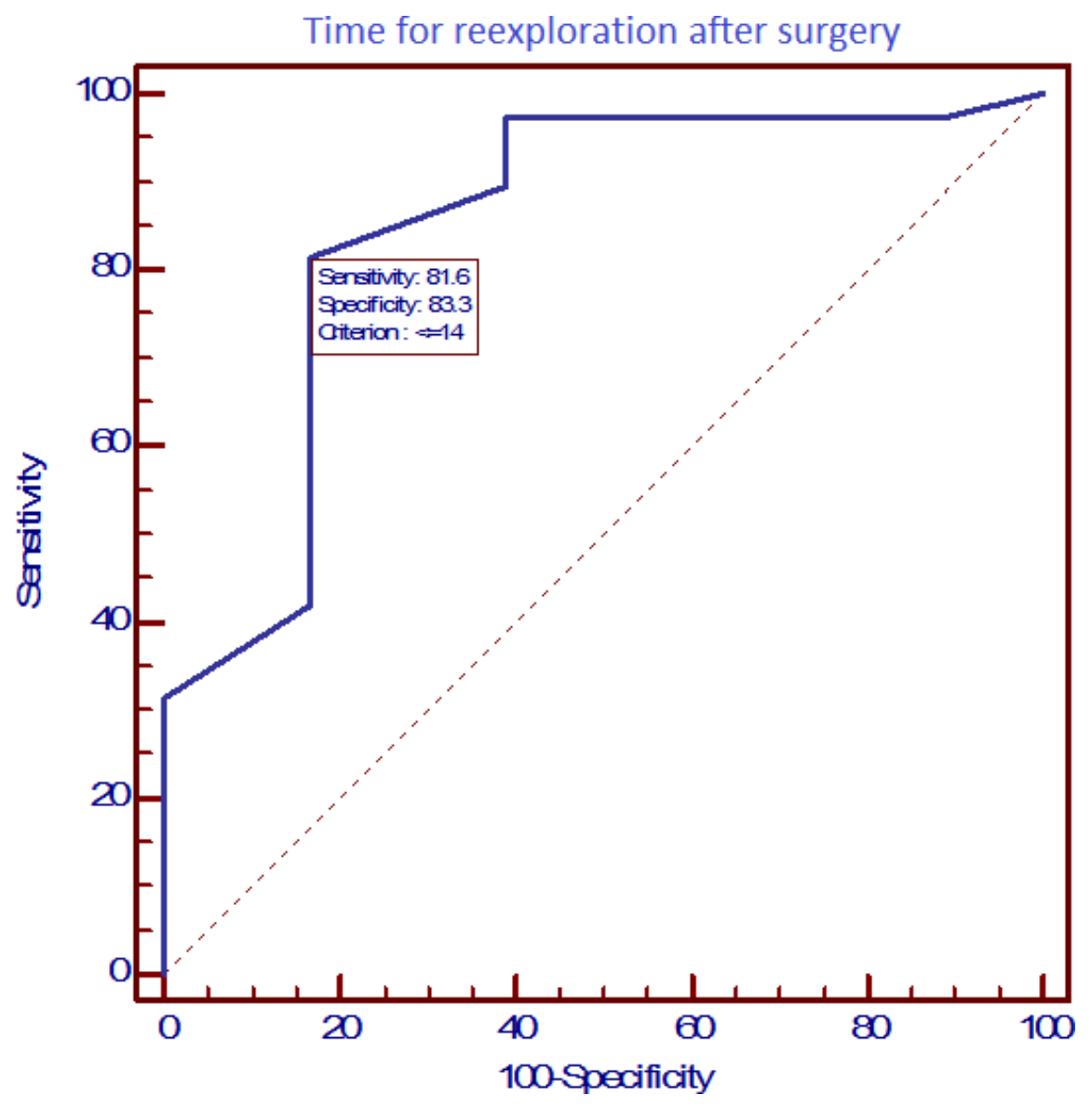

\section{Hosted file}

Table.docx available at https://authorea.com/users/336113/articles/461893-re-explorationafter-off-pump-coronary-artery-bypass-grafting-incidence-risk-factors-and-impact-oftiming 